\title{
Wired Core Network for Local and Premises Wireless Networks
}

\author{
Jiri Vodrazka', Tomas Hubeny ${ }^{1}$ \\ 1 Czech Technical University of Prague, Faculty of Electrical Engineering, \\ Technicka 2, 16627 Prague 6, Czech Republic \\ \{vodrazka, hubenyt\}@fel.cvut.cz
}

\begin{abstract}
The local and premises wireless networks have been expanding into many areas. The core network access point or basic station infrastructure can be made on wireless systems, on fixed cable systems with metallic twisted pairs, and, prospectively with optic fibers. The VDSL2 line systems with VDMT modulation and line bounding will be used for a data speed of hundreds $\mathrm{Mb} / \mathrm{s}$ and distance between active nodes can be measured in hundreds meters. A suggestion of active access network and simulation of data bit rate is presented.
\end{abstract}

Keywords: Core network, Active access network, VDSL2, VDMT modulation

\section{Introduction}

The backbone or core network access point or basic station infrastructure can be made on wireless systems, or on fixed cable systems with metallic twisted pairs and prospectively with optic fibers. Optical fibers are gradually replacing the metallic lines, and data bit rates have increased from tens $\mathrm{Mb} / \mathrm{s}$ to hundreds $\mathrm{Mb} / \mathrm{s}$ or $\mathrm{Gb} / \mathrm{s}$ in LANs and in the access network (first miles of digital subscriber lines). However, the information capacity of metallic lines can be increased if the systems use a VDSL2 [8], physical layer with vectored DMT modulation and line bounding concept. The methods for partial crosstalk cancellation are discussed and simulation results are presented for the middle-range used for connection of access points. The data bit rates $100 \mathrm{Mb} / \mathrm{s}$ and $1 \mathrm{~Gb} / \mathrm{s}$ are currently used for interfaces and connection to backbone network.

\section{Conception of core network}

If it is necessary to cover some area by high-speed wireless technology [6], the connection of access points to the central point of the network gateway has to be set. For connection of the access points sufficient throughput is necessary and hence the reason for building an efficient core network arises.

Wireless point-to-point or fixed point-to-multipoint connections are used for attachment of access points. This solution is not suitable under all circumstances. There can be problems with low throughput, interferences and insufficient bandwidth.

Please use the following format when citing this chapter:

Vodrazka, J., Hubeny, T., 2007, in IFIP International Federation for Information Processing, Volume 245, Personal

Wireless Communications, eds. Simak, B., Bestak, R., Kozowska, E., (Boston: Springer), pp. 332-340. 
For extreme throughput of the core network a fiber optic FTTB (Fiber to the Building), or FTTN (Fiber to the Node) FTTN conception can be used (Node is a wireless access point AP - see fig. 1). Here the length of the cables is restricted for common low-coast interfaces to $10-20 \mathrm{~km}$, which is more than satisfactory and also the transmission speed $1 \mathrm{~Gb} / \mathrm{s}$, eventually $10 \mathrm{~Gb} / \mathrm{s}$, offers a large reserve.

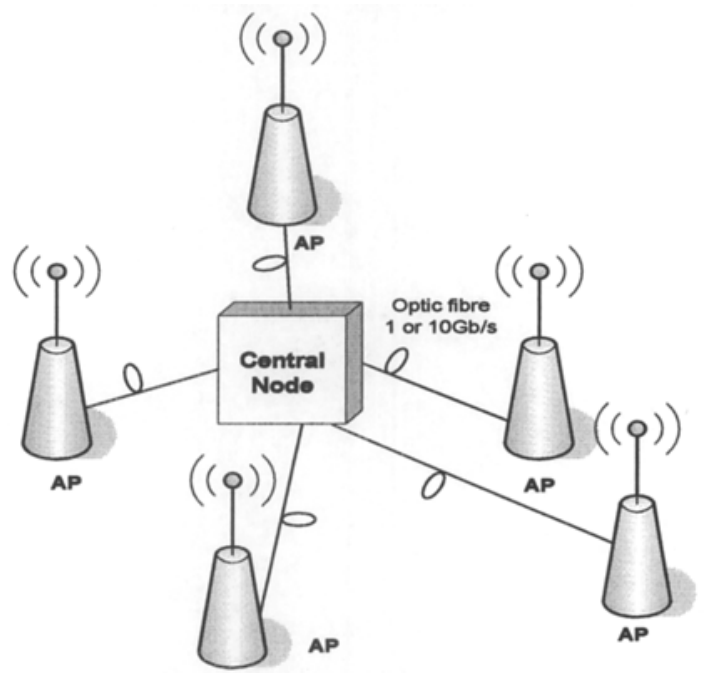

Fig. 1. The conception of hybrid optic-wireless FTTN network for core of wireless network.

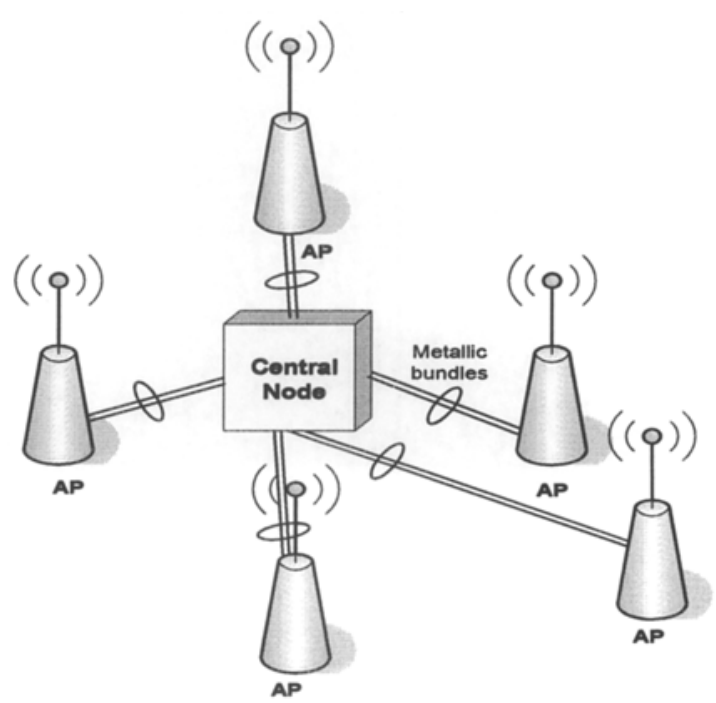

Fig. 2. The conception of wired core network for connection of wireless access points. 
The installation of new optic fiber infrastructure is not profitable anytime. On the other hand, classic metal cabling in the LAN networks has a restricted range of 100 $\mathrm{m}$. The solution is to use a VDSL2 physical layer with multi-pair bounding (see fig. 2) taking existing lines (e.g. telephone lines) into account. The metallic line provides speeds up to $100 \mathrm{Mb} / \mathrm{s}$. Using Multi-pair bounding which is working on the Inverse Multiplexing Principle will result in multiplying the transfer speed, hence it is possible to achieve a transmission speed to $1 \mathrm{~Gb} / \mathrm{s}$.

A problem in multi-pair cables is crosstalk between pairs, which is the main source of noise and affects the transfer speed. This can be solved by echo cancellation (section 2.1 and 2.2). Nevertheless, the transfer speed is only $1 \mathrm{~Gb} / \mathrm{s}$ for a distance of $500 \mathrm{~m}$ (for 10 pair bounding - section 3.3). In this case it is possible to move from a star to a tree topology and use a Hierarchic active core network concept (see fig. 3) on Hierarchic Active Access Network (HAAN). Total coverage for $1 \mathrm{~Gb} / \mathrm{s}$ will increase to a radius, only the lengths of the tree sections are restricted (approximately $500 \mathrm{~m}$ for local cable with diameter $0.4 \mathrm{~mm}$ ).

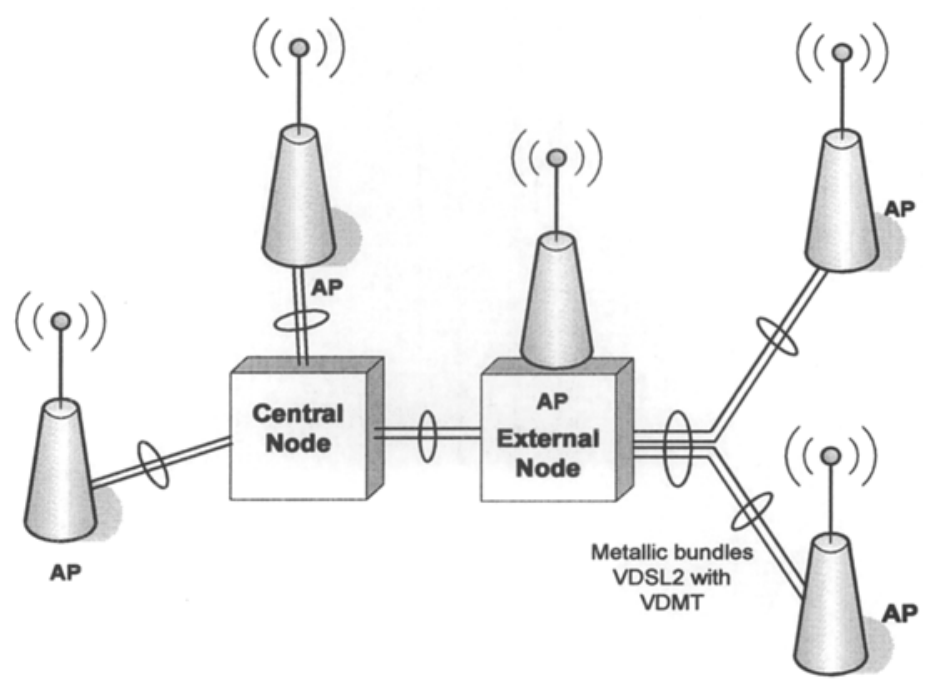

Fig. 3. The conception of wired Hierarchic active core network with VDSL2 (VDMT) for connection of wireless access points.

\subsection{Crosstalk cancellation}

A method of crosstalk cancellation is used in the GbE interface for cables of the $5 \mathrm{E}$ category for distances up to $100 \mathrm{~m}$. For further distance it is suitable to dispose of crosstalk at the near end using FDD frequency duplex and then deal with cancellation at the far end (see fig. 4). DMT very effectively cancels crosstalk, where cancellation between sub-channels is used. This modulation is then called vectored DMT (VDMT) and can be classed into a group of MIMO systems. (Multiple Input - Multiple Output) [10], [11]. 


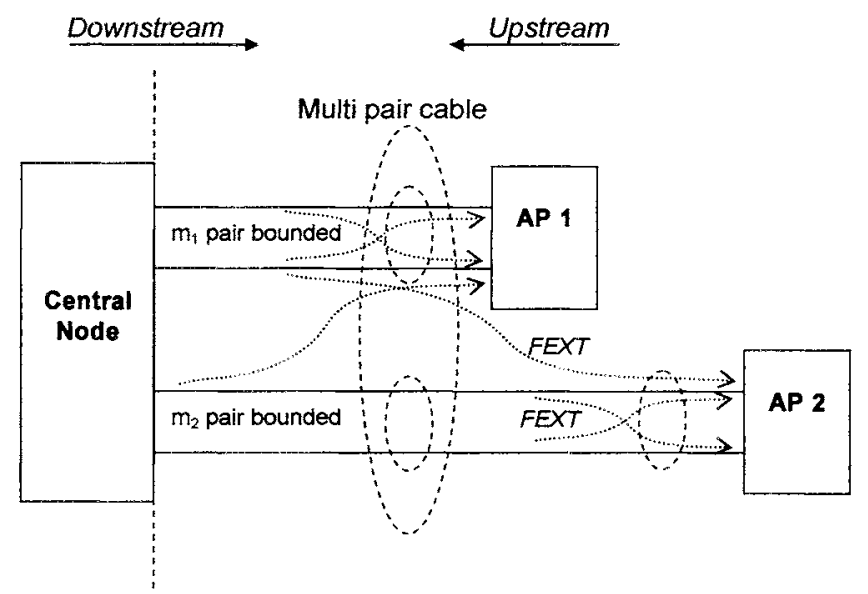

Fig. 4. The FEXT crosstalk for downstream direction in multi pair cable with pair bounding. The crosstalk exists between bounded pairs and between pairs from other bounding groups too.

\section{Central Node}

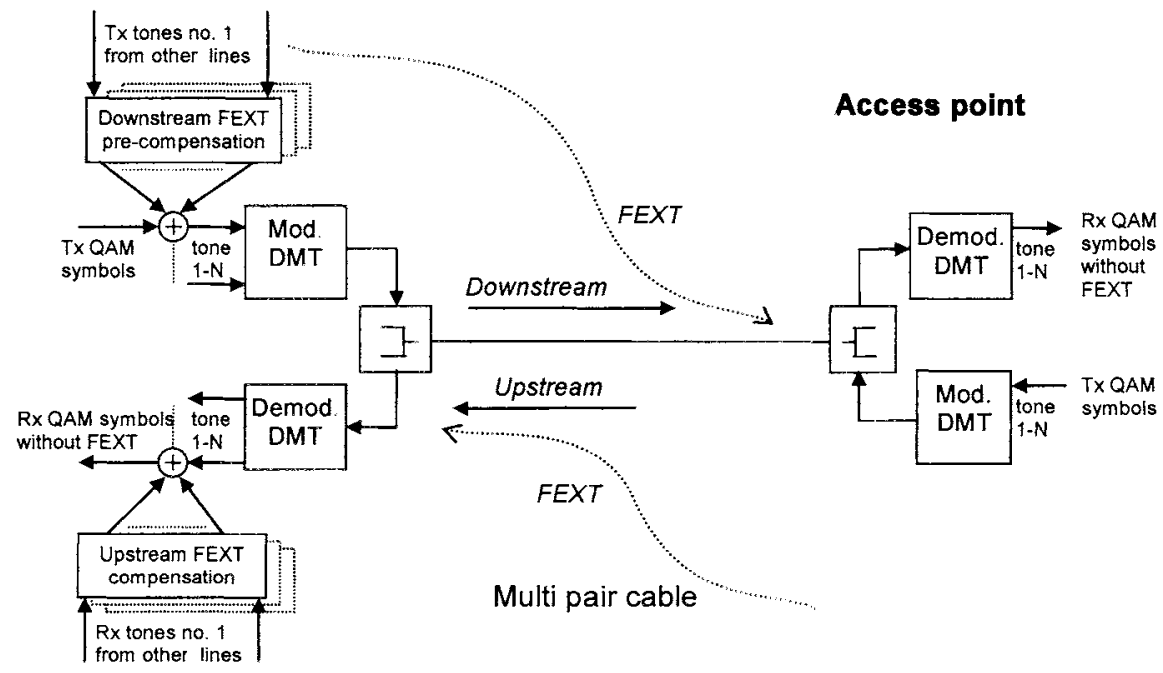

Fig. 5. The principle of VDMT for multi pair cable - FEXT compensation on receiver side $(\mathrm{Rx})$ in frequency domain for upstream and pre-compensation on transceiver side $(\mathrm{Tx})$ in frequency domain for downstream.

The advantage of VDMT is a possibility to create multipoint networks, where the cancellation for both directions is done in a central network element. For the upstream 
direction there is compensation at the receiver side and for downstream there is precompensation at the transmitter side (fig. 5).

For correct operation of VDMT it is necessary to know the transmission path parameters, along with the nature of crosstalk from surrounding (neighboring) lines, placed in identical metallic cables. Transmission path parameters [9] are identified during the process of establishing connection between access points and central node. To compensate for crosstalk it is required to have signals sent from all lines. They are present in central mode, but not in each access point. Therefore, compensation has to be performed in both direction of transmission in central node, or more precisely for direction upstream crosstalk compensation on receiving side and for direction downstream signal's pre-compensation on transmitter side.

\subsection{Hierarchical active core network with VDMT}

Conception of a hierarchic active access network solves the problem of markedly restricted distance when the extreme transmission speeds are required. The increased reach is possible using active elements in cable tree structure analogous to a hybrid access network, FTTC. The only difference is that the active element is not connected to the access point via an optic fiber but via metallic wire bundles using inverse multiplex principle (IM)

For maximization of the transfer speed usage of crosstalk cancellation in a bundle of 10 pairs ( 5 quads) is suitable. In local branching there are access points as well as active elements of core network. In active network elements the multiplexing of data from users is accomplished.

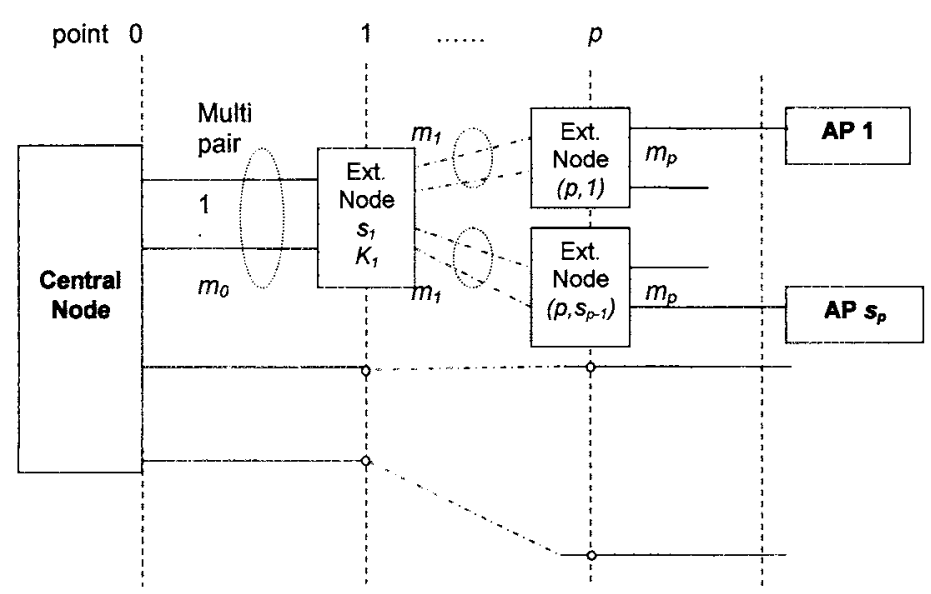

Fig. 6. The structure of hierarchic active core network for connection access points (AP) to central node trough external nodes (can include access points too) with p-levels.

In general, layout of the access network is figured out with a structure (see fig. 6). The central node is connected via bundle of $m_{0}$ pairs to the external node. Transmission in the bundle is ensured by VDSL2 subscriber lines with VDMT modulation. Supposing 
identical transfer speeds of all lines which operate at distance of $d_{0}$ in the bundle $R\left(\mathrm{~d}_{0}\right)$ the resulting transfer speed of this section is $R_{0}=m_{0} . R\left(\mathrm{~d}_{0}\right)$. With this speed the external node is connected to the central node.

Users can be connected via external DSLAM or another hierarchic level of external DSLAMS can follow. Supposing the number of external DSLAM's $s_{1}$, connected with the number of pairs $m_{l}$ at distance $d_{l}$ the speed is $R_{l}=m_{l}, R\left(\mathrm{~d}_{1}\right)$. The traffic concentration is used, hence $R_{0} \leq s_{l} R_{l}$. The $K_{I}$ concentration factor is established, so it can be expressed:

$R_{0}=R_{1} \frac{s_{1}}{K_{1}}$ and generally for $p$ levels of hierarchy $R_{0}=R_{p} \prod_{i=1}^{p} \frac{s_{i}}{K_{i}}$

Followed by:

$m_{0} R\left(d_{0}\right)=m_{p} R\left(d_{p}\right) \cdot \prod_{i=1}^{p} \frac{s_{i}}{K_{i}}$

Assuming the speed at the distance of $p$ :

$R_{p}=m_{0} R\left(d_{0}\right) \cdot \prod_{i=1}^{p} \frac{K_{i}}{s_{i}}$

The resulting speed for the user will be limited by throughput of all sections:

$$
R_{s}=\min \left\{m_{p} R_{p}\left(d_{p}\right), m_{p-1} R_{p-1}\left(d_{p-1}\right) \cdot \frac{K_{p}}{s_{p}} ; \ldots ; m_{1} R_{\mathrm{I}}\left(d_{1}\right) \cdot \prod_{i=2}^{p} \frac{K_{i}}{s_{i}} ; m_{0} R_{0}\left(d_{0}\right) \cdot \prod_{i=1}^{p} \frac{K_{i}}{s_{i}}\right\}
$$

For the practical applications it is not suitable to create more than two hierarchy levels. The concentration rate can be chosen, in ranges according to types of provided services, e.g. 1: $K_{1}$, where $K_{1}$ is from interval 2-20 at one level hierarchy, eventually $1: \mathrm{K}_{1}: \mathrm{K}_{2}$ where $\mathrm{K}_{1}$ is from interval $2-5$ and $\mathrm{K}_{2}$ is from interval $2-8$ at two level hierarchy.

\section{Simulation results}

\subsection{Simulation of transmission environment}

Metallic lines in local networks begin at the main distribution frame, then they run as multi-pair cables to line distribution frames, from which they branch into smaller groups of subscriber lines or to individual lines leading to the subscribers' premises. In this network there are various types of cables in common use with copper core (mostly $0.4 \mathrm{~mm}$ in diameter) and various numbers of pairs, basically arranged in quads. The principal factors limiting the transmission of high-speed signals are attenuation of the line, and crosstalk between the individual pairs [3], [4]. 
Since there is a need to estimate the available transmission speed, we have used a MATLAB Web Server to design a simulator of XDSL lines that is available at our web pages [1]. The program accepts many input parameters for the calculation and provides results in various forms. The most important simulation result is the estimated transmission speed for upstream and especially for downstream direction.

\subsection{Crosstalk modeling}

The described method for summarizing of contributive crosstalk (NEXT and FEXT) has been recommended by the FSAN (Full Service Access Network) consortium. The simplification of crosstalk computation consists in crosstalk parameters. These parameters are averaged over the total length of the subscriber line, not considering the real cascade structure. In addition, the position (in the same group, in different groups) of disturbing and disturbed pairs is ignored. The values of crosstalk constants are preventively calculated for the worst-case disturbance environment scenario. However, this worst case results in a more pessimistic noise level.

The typical attribute of crosstalk is very high variance of values. It is clear that if we interleave the minimum attenuation values, we obtain the worst-case disturbance scenario, which is approximately $10 \mathrm{~dB}$ worse than average attenuation values. The maximum attenuation values are 12 or $15 \mathrm{~dB}$ (or more) higher then the worst-case scenario [5].

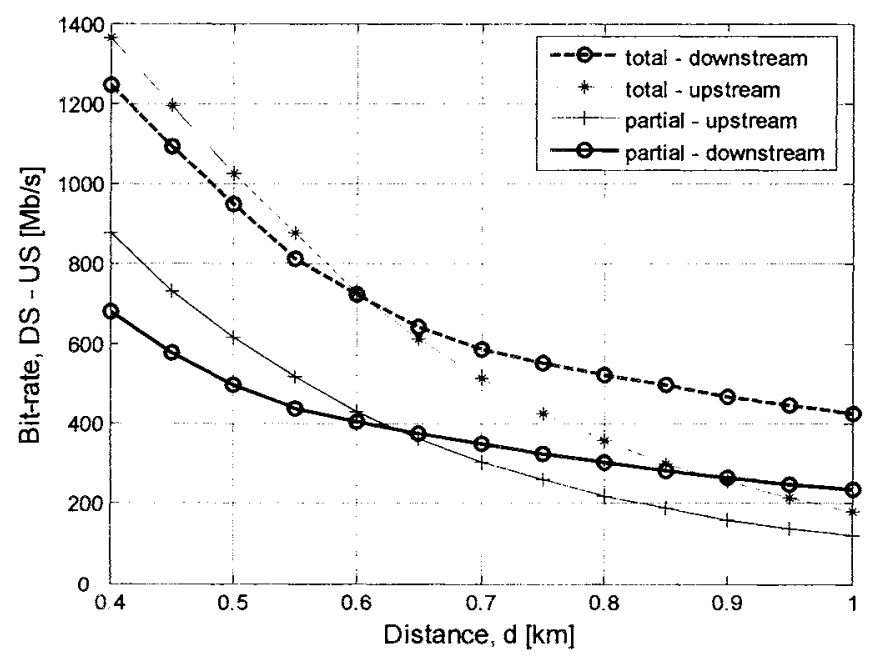

Fig. 7. Total transmission speeds of a bundle for 997 plan with full (dash line) and partial (full line) VDMT coordination.

The frequency dependence of crosstalk transmission function has a random character. With respect to the construction of standard cables used in access networks, it is possible to divide the symmetric pairs into several groups. Each group shall contain pairs with the same position. The knowledge of crosstalk transfer function between all pairs will be necessary for transmission using Vectored DMT modulation. 


\subsection{Results for hierarchic active core network}

For estimation of reachable transfer speed let's assume that crosstalk cancellation is used in a bundle with 10 pairs ( 5 quads). Hence let's focus on estimation of transfer speed reachable in the bundle of ten lines. Supposing total FEXT cancellation is used, a residual noise AWGN will take effect only $(-130 \mathrm{dBm} / \mathrm{Hz})$. Then the downstream and upstream speeds will occur - as shown in fig. 7 (frequency plan 997) and fig. 8 (frequency plan 998).

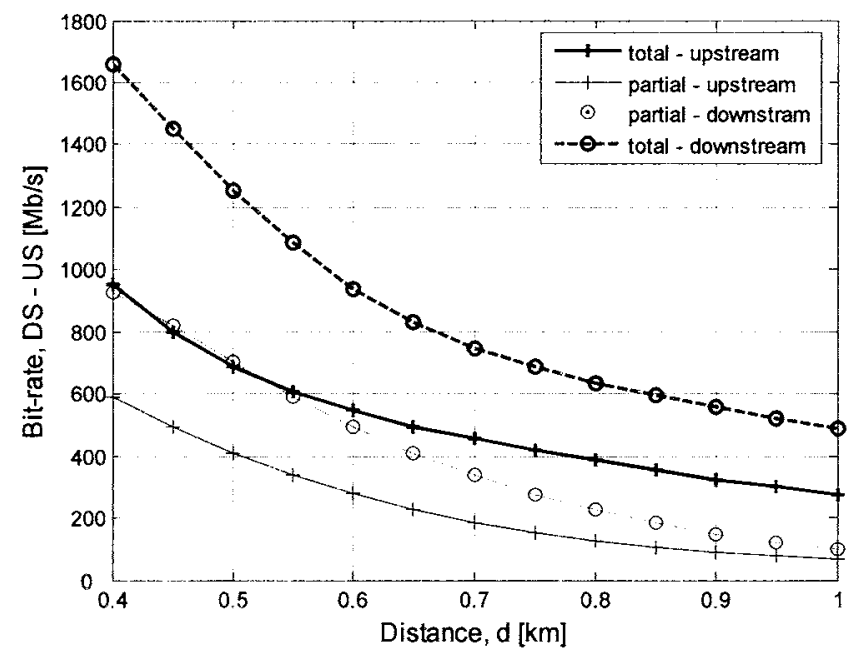

Fig. 8. Total transmission speeds of a bundle for 998 plan with full (dash line) and partial (full line) VDMT coordination.

Practically, in a cable other subscriber's lines will be present - ADSL (assumed 20\% from the cable profile), ADSL2+ (assumed $10 \%$ from the cable profile) and SHDSL (assumed $6 \%$ from the cable profile). Hence slower transfer speeds will occur - in figures described by the full line.

\section{Conclusion}

Existing metallic cables which were originally designated for low-frequency telephone transmissions are used for digital transmission with speeds up to hundreds $\mathrm{Mb} / \mathrm{s}$. In the paper, the application of these systems for core network of access points was shown. Using VDMT modulation brings a significant increase in transfer speed in cables which are already filled with other subscriber's line in comparison to common way of transmission. This places considerable demand on the computation capacity of signal processors. The solution is to use a partial crosstalk cancellation with a selection of the most interfering DMT tones. According to our experiments this method does not have the results as we expected and hoped. 
Nevertheless for transmission within medium distances with a bundle of lines, speeds up to $1 \mathrm{~Gb} / \mathrm{s}$ can be reach using a physical layer VDSL2 (plan 997). Therefore transmission among network elements in core network for wireless access points can be performed thus.

The active core network on a basic Hierarchic Active Access Network (HAAN) was performed. Global coverage for speeds of $1 \mathrm{~Gb} / \mathrm{s}$ will differ according to the number of hierarchical levels. The lengths of the cables are restricted to $500 \mathrm{~m}$ for cables with wire diameter of $0.4 \mathrm{~mm}$, as follows from the results of simulations. For extreme transfer speeds the metallic infrastructure has to be replaced by an optic fiber.

Acknowledgments: This paper has originated thanks to the support of the Grant Agency of the Czech Republic within project GA102/07/1503 and Czech Technical University's grant No. CTU0715013.

\section{References:}

1. Vodrazka, J. - Jares, P. - Hubeny, T.: xDSL simulator. Matlab Server on-line. http://matlab.feld.cvut.cz/en/

2. Vodrazka, J.: Downstream Power-Back-Off Used for ADSL. EC-SIP-M 2005. Bratislava: Slovak University of Technology, 2005, pp. 349-353. ISBN 80-227-2257-X

3. Vodrazka, J. - Simak, B.: Theoretical Limits of DSL Lines. Communication and Information Technologies - Conference Proceedings. Liptovský Mikuláš, 2005, pp. 243246. ISBN 80-8040-269-8

4. Simak, B. - Vodrazka, J.: Limits for Broadband Transmission on the Twisted Pairs and Other System Co-existence. CTU Workshop. Prague: CTU 2006

5. Jares, P.: Throughput Modelling and Spectral Compatibility of Digital Subscriber Lines. EC-SIP-M 2005. Bratislava: The Faculty of Electrical Engineering and Information Technology of the Slovak University, 2005, s. 228-233. ISBN 80-227-2257-X.

6. Konhäuser, W.: Broadband Wireless Access Solutions - Progressive Challenges and Potential Value of Next Generation Mobile Networks. Wireless Personal Communications: An International Journal archive. Kluwer Academic Publishers Hingham, MA, USA. Volume 37, Issue 3-4 (May 2006). Pages: 243 - 259 ISSN:0929-6212

7. Tardy, I. - Bråten, L.E. - Bichot, G. - Settembre, M....: Hybrid architecture to achieve true broadband access in rural areas. BroadBand Europe Brugge, BELGIUM 08-10 December 2004. Session 07 - Paper 07-02. Page 1 of 6.

8. Very-high-speed Digital Subscriber Line (VDSL2). ITU-T Recommendation G.993.2

9. Rauschmayer, D. J.: ADSL/VDSL Principles: A Practical and Precise Study of Asymmetric Digital Subscriber Lines and Very High Speed Digital Subscriber Lines. Indianapolis, USA: Macmillan Technical Publishing, 1999

10. Cendrillona, R. - Ginisb, G. - Moonena, M. - Acker, K.: Partial Crosstalk Precompensation in Downstream VDSL. Signal Processing 84. Elsevier (2004), pp. 20052019

11. Brady, M. H. - Cioffi, J. M.: The Worst-Case Interference in DSL Systems Employing Dynamic Spectrum Management. Hindawi Publishing Corporation EURASIP Journal on Applied Signal Processing. Volume 2006, Article ID 78524, pp. 1-11. 